\title{
Dark Matter Protohalos in MSSM-9 and Implications for Direct and Indirect Detections
}

\author{
Roberta Diamanti ${ }^{* \dagger}$ \\ GRAPPA Institute, University of Amsterdam, 1098 XH Amsterdam, The Netherlands \\ E-mail: r.diamanti@uva.nl
}

\begin{abstract}
We study how the kinetic decoupling of dark matter within a minimal supersymmetric extension of the standard model, by adopting nine independent parameters (MSSM-9), could improve our knowledge of the properties of the dark matter protohalos. We show that the most probable neutralino mass regions, which satisfy the relic density and the Higgs mass contraints, are those with the lightest supersymmetric neutralino mass around $1 \mathrm{TeV}$ and $3 \mathrm{TeV}$, corresponding to Higgsino-like and Wino-like neutralino, respectively. The kinetic decoupling temperature in the MSSM-9 scenario leads to a most probable protohalo mass in a range of $M_{\mathrm{ph}} \sim 10^{-12}-10^{-7} M_{\odot}$. The part of the region closer to $\sim 2 \mathrm{TeV}$ gives also important contributions from the neutralinostau co-annihilation, reducing the effective annihilation rate in the early Universe. We also study how the size of the smallest dark matter substructures correlates to experimental signatures, such as the spin-dependent and spin-independent scattering cross sections, relevant for direct detection of dark matter. Improvements on the spin-independent sensitivity might reduce the most probable range of the protohalo mass between $\sim 10^{-9} M_{\odot}$ and $\sim 10^{-7} M_{\odot}$, while the expected spin-dependent sensitivity provides weaker constraints. We show how the boost of the luminosity due to dark matter annihilation increases, depending on the protohalo mass. In the Higgsino case, the protohalo mass is lower than the canonical value often used in the literature $\left(\sim 10^{-6} M_{\odot}\right)$, while $\langle\sigma v\rangle$ does not deviate from $\langle\sigma v\rangle \sim 10^{-26} \mathrm{~cm}^{3} \mathrm{~s}^{-1}$; there is no significant enhancement of the luminosity. On the contrary, in the Wino case, the protohalo mass is even lighter, and $\langle\sigma v\rangle$ is two orders of magnitude larger; as its consequence, we see a substantial enhancement of the luminosity.
\end{abstract}

The European Physical Society Conference on High Energy Physics

22-29 July 2015

Vienna, Austria

\footnotetext{
* Speaker.

${ }^{\dagger}$ Poster presenter at The European Physical Society Conference on High Energy Physics 2015 in Vienna.
} 


\section{Introduction}

We do a forecast on the mass of the protohalos, $M_{\mathrm{ph}}$, within a supersymmetric scenario by taking into account the latest data from all the relevant particle physics experiments as well as the relic density constraints. We perform our analyses within a Bayesian framework, and obtain maps of the probability densities of different regions of the parameter space. Recentely, authors found a strong correlation between the kinetic decoupling temperature and the spin dependent (SD) cross section of neutralinos off nucleons; on the contrary, a weaker correlation was found in the case of the spin independet (SI) neutralino-nucleon cross section. In the most probable cases, we find that $M_{\mathrm{ph}}$ correlates with both SD and SI scattering cross sections. We show how future direct detection experiments can play an important role in constraining the (most probable) minimal $M_{\mathrm{ph}}$ down to $10^{9} \mathrm{M}_{\odot}$. We also show the changes of the expected value of the boost of the luminosity due to the annihilation of dark matter in those regions.

\section{Analysis}

To study the MSSM parameter space we perform a Bayesian analysis. We assume gravity mediated SUSY breaking and parameterize the MSSM with 10 fundamental parameters defined at the unification scale of the gauge couplings as well as SM parameters, among which 9 of them we allow to vary after requiring the correct electroweak symmetry breaking. We also assume unification and universality conditions for the squark masses, slepton masses and trilinear terms. The set of 10 parameters is well described in Ref. [1].

To perform the analysis, we consider two different priors: standard log priors (S-log prior), which takes a log prior for each parameter independently, and improved log priors (I-log prior), which assumes a common origin for the soft-masses, as expected from SUSY breaking mechanisms. The range of the parameters in our scan varies from $10 \mathrm{GeV}$ to $10^{6} \mathrm{GeV}$.

The experimental data considered in our analysis is described in Table 2 of Ref. [1], where we include electroweak precision measurements [2], B-physics observables [3] , the Higgs mass [4], and constraints on the WIMP-nucleon scattering cross-section by XENON-100 [5]. In addition, we include the measured relic density according to Planck results [6] because we assume a scenario with a single DM component which is produced thermally in the early Universe. ${ }^{1}$

For the numerical analysis we use SuperBayeS-v2.0, a publicly available package that include MultiNest [8] nested sampling algorithm, Softsusy [9] for the computation of the mass spectrum, micromegas [10] for the computation of the relic density, Darksusy [11] for the computation of direct ${ }^{2}$ and indirect detection observable, SusyBSG [13] and Superiso [14] for B-physics observable.

For the Wino-like and Higgsino-like LSP cases, the Sommerfeld enhancement of the primordial

\footnotetext{
${ }^{1}$ In our analysis, we assume that $100 \%$ of dark matter consists of the neutralino. If there is other dark matter components, we need to regard the measurement of the dark matter density determined by Planck satellite as an upper limit, and follow some scaling ansaz studied in, e.g., [7].

${ }^{2}$ For the contribution of the light quarks to the nucleon form factors, concerning the spin-independent WIMPnucleon cross section, we have adopted the values $f_{T u}=0.02698, f_{T d}=0.03906$ and $f_{T s}=0.36$ [12], derived experimentally from measurements of the pion-nucleon sigma term.
} 
and present day neutralino annihilation has been included, using DarkSE [15], which is a package for DarkSusy. We created a grid in the $M_{2}-\mu$ plane and performed interpolations to correct the values of the relic density and the present day neutralino annihilation within SuperBayes interface.

\section{The most probable regions and inplications for Direct and Indirect Detection}

The determination of the smallest mass of the DM protohalo for the most probable regions of the MSSM is of great interest for the study of both direct and indirect detection of DM.

Both Higgs mass measurement and relic density constraint are the main responsible for the shift of the preferred regions towards higher masses.
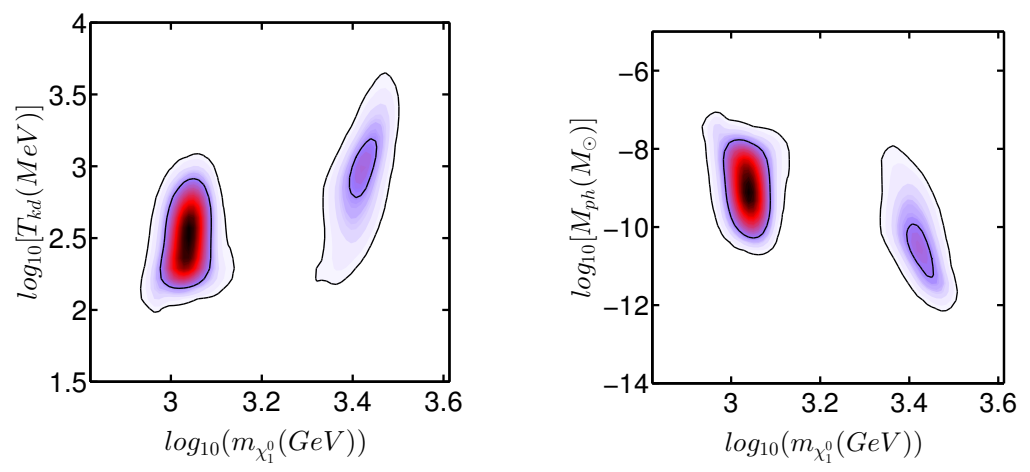

Figure 1: The two dimensonal joint posterior probability density function for the temperature of kinetic decoupling, $T_{k d}$, versus the neutralino mass (left panel), and for the protohalo mass, $M_{\mathrm{ph}}$, versus the neutralino mass (right panel). The region with higher probability density corresponds to a Higgsino DM candidate, while in the second region the DM candidate is a Wino.

In Fig. 1 we show the two dimensional joint posterior PDF for the temperature of kinetic decoupling, $T_{k d}$, and for protohalo mass, $M_{\mathrm{ph}}$, against the neutralino mass. The contours represent intervals at $68 \%$ and $95 \%$ credible regions. The two most probable regions are around $\sim 1 \mathrm{TeV}$ and $\sim 3 \mathrm{TeV}$ and correspond to Higgsino-like and Wino-like neutralino, respectively.

\subsection{Implication for Direct Detection}

Figure 2 shows points that reproduce the experimental constraint at $2 \sigma$ confidence level for the minimal protohalo mass versus the tree level SD cross section plane. The right panel shows the case where the lightest first or second generation of sfermions is at least nine times heavier that the lightest neutralino, $\Delta\left(m_{\tilde{l} \tilde{q}}-m_{\chi_{1}^{0}}\right)>0.8$. The thin yellow line corresponds to $\sim 1 \mathrm{TeV}$ Higgsino-like neutralino, while the thin red line to $\sim 3 \mathrm{TeV}$ Wino-like neutralino. In these two cases the Z-boson mediates both scattering processes. The rest of the points correspond to the Bino-like neutralino where, instead of a line, we get scattered points with $100 \mathrm{GeV} \lesssim m_{\chi_{1}^{0}} \lesssim 1 \mathrm{TeV}$.

Regarding $\sigma_{p}^{\mathrm{SD}}$ for the Bino-like region, the dominant process is mediated by the Z-boson. ${ }^{3}$ Besides

\footnotetext{
${ }^{3}$ Squarks are typically heavier than sleptons when parameterizing the model at gauge coupling unification scale. Therefore, imposing the condition $\Delta\left(m_{\tilde{l}}-m_{\chi_{1}^{0}}\right)>0.8$ implies that squarks are typically much heavier than ten times the mass of the lightest neutralino.
} 


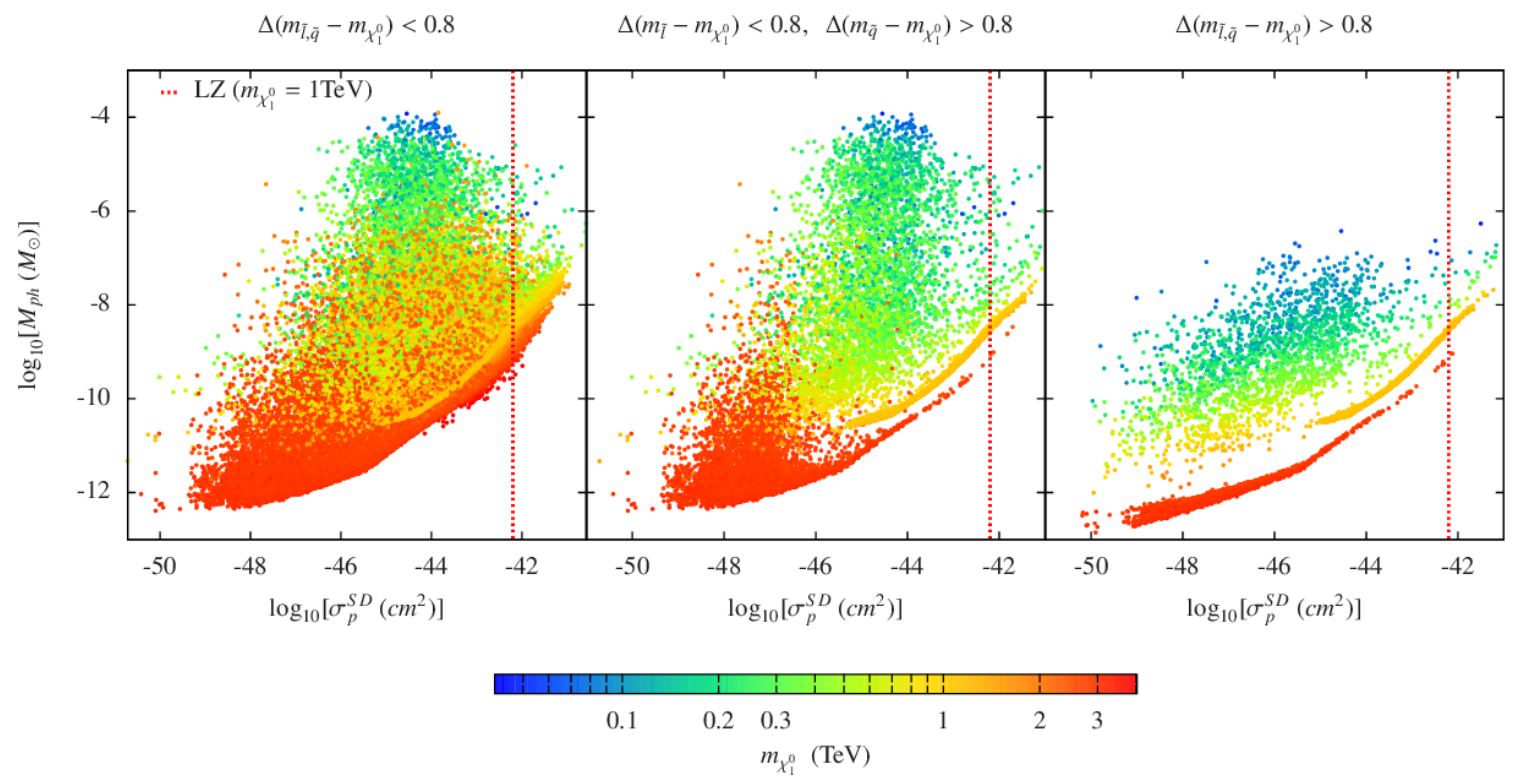

Figure 2: Points that reproduce all the experimental observables at $2 \sigma$ confidence level in the SD cross section $\sigma_{p}^{S D}$ versus protohalo mass $M_{\mathrm{ph}}$ plane. The neutralino mass is indicated with colors, as shown in the color bar. The three panels separate the points in three groups: light squarks and sleptons (left panel), light sleptons and decoupled squarks (central panel), and decoupled squarks and sleptons (right panel).

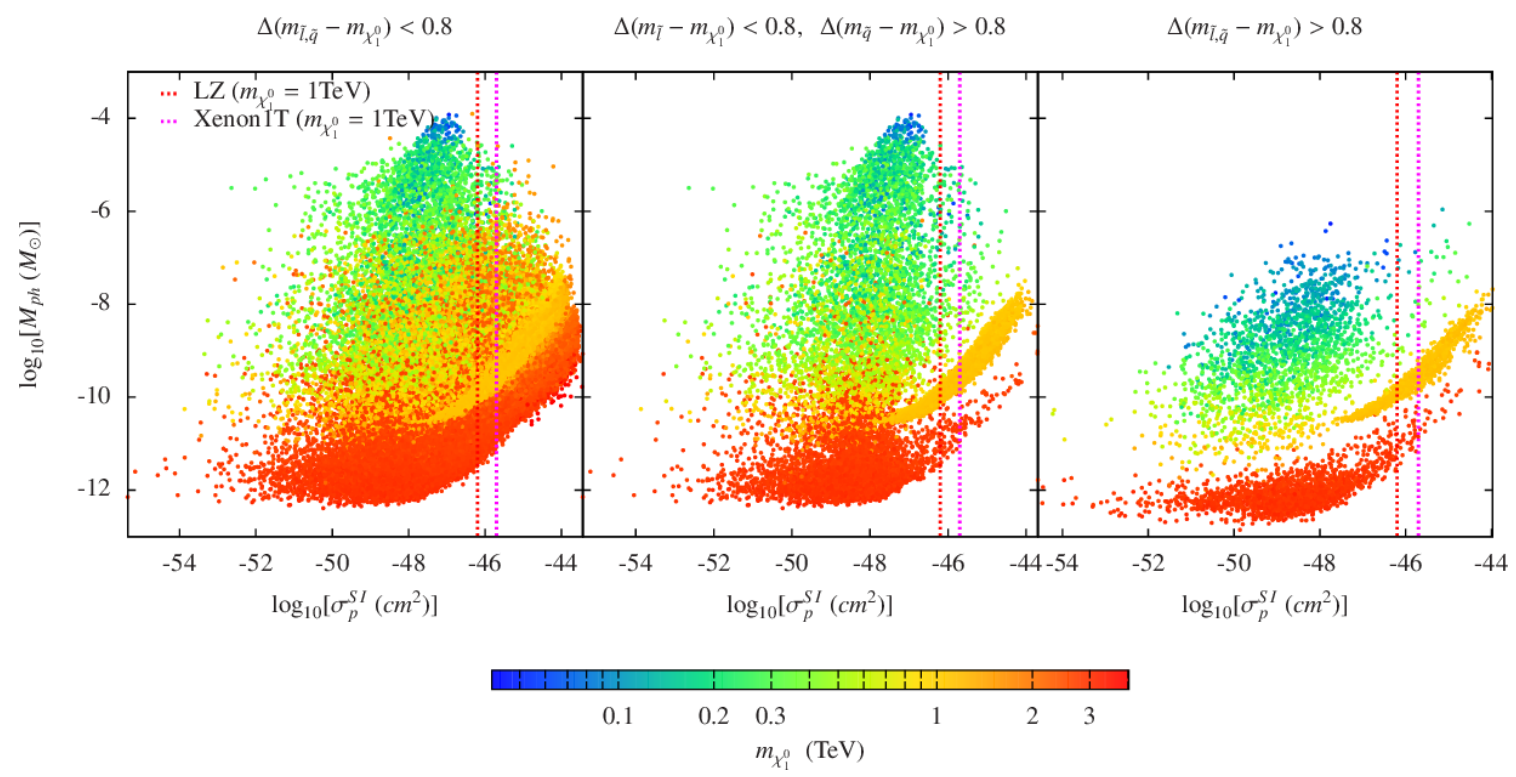

Figure 3: Same as Fig.2 for the SI cross section, $\sigma^{S I}$.

the dominant scattering processes for $T_{k d}$ and $\sigma_{p}^{\mathrm{SD}}$ are different, there is an apparent correlation between the two quantities for a fixed neutralino mass.

Central panel of Fig. 2 shows the case where the lightest slepton has a mass smaller than $\sim 10$ times the lightest neutralino mass. As expected, the Wino-like and Bino-like regions spread to larger protohalo masses. 
Left panel of Fig. 2 shows the case where the lightest sleptons and squarks are smaller than $\sim 10$ times the lightest neutralino. Here, squarks are light enough to give important contributions to the scattering with the nucleus, spreading the points to larger values of $\sigma_{n}^{S D}$.

Figure 3.1 shows points in the minimal protohalo mass versus tree level SI cross section plane. The main contribution to the SI cross section comes from the Higgs exchange, requiring a nonnegligible Higgino and Wino/Bino coupling (since Higgs couplings through neutralinos are $H \tilde{H} \tilde{B}$ and $H \tilde{H} \tilde{W}$ ). On the other hand, the total neutralino-SM scattering, and therefore $T_{k d}$ and $M_{\mathrm{ph}}$, are dominated by SD interactions. A a consequence, right panel of Fig. 3.1 shows the correlation between the $Z \chi_{1}^{0} \chi_{1}^{0}$ and $H \chi_{1}^{0} \chi_{1}^{0}$, for the Higgsino and Wino case. Central and left panels show the effect of sleptons and squarks in the scattering processes.

Figures 2 and 3.1 show the expected sensitivity by Xenon1T and LZ assuming the neutralino mass is $\sim 1 \mathrm{TeV}$. For a neutralino of $\sim 100 \mathrm{GeV}$, the expected sensitivity is around one order of magnitude stronger.

\subsection{Implications for Indirect detection}

Another way to look for dark matter is through indirect detection methods, which consist to detect, indirectly, the lightest supersymmetric particle through annihilation processes where SM particles, including gamma-ray photons, are produced. Since the luminosity of each subhalo in the host halo due to the dark matter annihilation processes depends on the volume integral of the subhalo density squared, smaller and denser substructures provide an enhancement of the luminosity. We show the ratio of the luminosity over the reference one $\tilde{L} \equiv L / L_{r e f}{ }^{4}$, versus the DM mass, $m_{\chi_{1}^{0}}$. We also analyzed the change in the boost by varying the $\gamma$-parameter ${ }^{5}$ in a range between 0.5 and 0.9 , we only show the case $\gamma=0.8$, and found that $\tilde{L}$ always got largely boosted by decreasing $\gamma$.
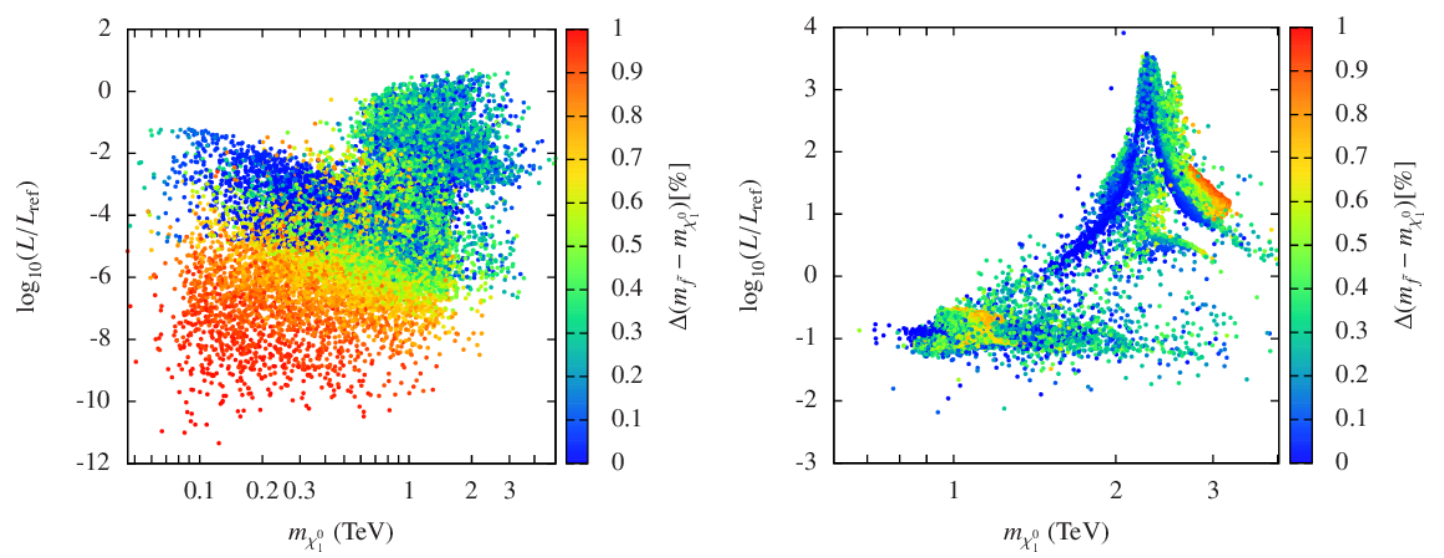

Figure 4: The mass of the lightest neutralino versus the boost factor, $\tilde{L} \equiv L / L_{r e f}$, for points that reproduce all the experimental observables within $2 \sigma$ confidence level. Left panel shows points which refer to a Binofraction $\left(N_{11}\right)$ larger than 0.8 . Right panel shows point with a Bino-fraction smaller than 0.8

\footnotetext{
${ }^{4}$ For further details about the luminosity we address the reader to see Ref.[1] and references therein. For values of the reference parameters, we adopt $\langle\sigma v\rangle_{\text {ref }}=3 \times 10^{-26} \mathrm{~cm}^{3} \mathrm{~s}^{-1}, M_{\text {ref }}=10^{-6} M_{\odot}$, and $\gamma=0.8$.

${ }^{5} \gamma$ is a phenomenological parameter describing the scaling behavior of the luminosity of each subhalo below the resolution of a N-body simulation. For further details, see [1].
} 
Figure 4 shows the boost factor, $\tilde{L} \equiv L / L_{r e f}$, for points that reproduce all the experimental observables within $2 \sigma$ confidence level. Right panel shows points which refer to a Higgisino-like and Wino-like neutralinos, while the left panel shows points where the neutralino is mostly Binolike. Bino-like neutralinos have very small $\langle\sigma v\rangle$ in the limit of zero velocity. Co-annihilations, which play a very important role in the efficient annihilation in the early Universe, are not present anymore; this is the reason for which we have a very small boost of the luminosity.

\section{References}

[1] R. Diamanti, M. E. C. Catalan and S. Ando, Dark matter protohalos in a nine parameter MSSM and implications for direct and indirect detection, Phys. Rev. D92 (2015), no. 6065029 [1506.01529].

[2] “The LEP Electroweak Working Group.” http://lepewwg.web.cern.ch/LEPEWWG.

[3] Heavy Flavor Averaging Group Collaboration, Y. Amhis et. al., Averages of B-Hadron, C-Hadron, and tau-lepton properties as of early 2012,1207.1158.

[4] ATLAS Collaboration Collaboration, G. Aad et. al., Observation of a new particle in the search for the Standard Model Higgs boson with the ATLAS detector at the LHC, Phys.Lett. B716 (2012) 1-29 [1207.7214].

[5] XENON100 Collaboration Collaboration, E. Aprile et. al., Dark Matter Results from 225 Live Days of XENON100 Data, Phys.Rev.Lett. 109 (2012) 181301 [1207.5988].

[6] Planck Collaboration, P. Ade et. al., Planck 2013 results. XVI. Cosmological parameters, Astron.Astrophys. 571 (2014) A16 [1303.5076].

[7] G. Bertone, D. G. Cerdeno, M. Fornasa, R. R. de Austri and R. Trotta, Identification of Dark Matter particles with LHC and direct detection data, Phys.Rev. D82 (2010) 055008 [10 05.4280$].$

[8] F. Feroz and M. P. Hobson, Multimodal nested sampling: an efficient and robust alternative to MCMC methods for astronomical data analysis, Mon. Not. Roy. Astron. Soc. 384 (2008) 449-463 [0704.3704].

[9] B. Allanach, SOFTSUSY: a program for calculating supersymmetric spectra, Comput.Phys.Commun. 143 (2002) 305-331 [hep-ph/ 0104145$].$

[10] G. Belanger, F. Boudjema, A. Pukhov and A. Semenov, MicrOMEGAs: A Program for calculating the relic density in the MSSM, Comput.Phys.Commun. 149 (2002) 103-120 [hep-ph/0112278].

[11] P. Gondolo, J. Edsjo, L. Bergstrom, P. Ullio and E. A. Baltz, DarkSUSY: A Numerical package for dark matter calculations in the MSSM, astro-ph/0012234.

[12] J. R. Ellis, K. A. Olive and C. Savage, Hadronic Uncertainties in the Elastic Scattering of Supersymmetric Dark Matter, Phys.Rev. D77 (2008) 065026 [0801. 3656].

[13] G. Degrassi, P. Gambino and P. Slavich, SusyBSG: A Fortran code for BR[B ->X(s) gamma] in the MSSM with Minimal Flavor Violation, Comput.Phys.Commun. 179 (2008) 759-771 [0712. 3265].

[14] F. Mahmoudi, SuperIso v2.3: A Program for calculating flavor physics observables in Supersymmetry, Comput.Phys.Commun. 180 (2009) 1579-1613 [0808 . 3144 ].

[15] A. Hryczuk, R. Iengo and P. Ullio, Relic densities including Sommerfeld enhancements in the MSSM, JHEP 1103 (2011) 069 [1010.2172]. 